\title{
Wie man Teilhabe steuern kann
}

\section{Ein Forschungsvorhaben in der Eingliederungshilfe sucht nach Kriterien für die Praxis}

\author{
HEIKE GOLLETZ \\ Heike Golletz ist Referentin für \\ Qualitätsmanagement in der \\ Bundesarbeitsgemeinschaft \\ der Freien Wohlfahrtspflege. \\ Heike.Golletz@bag-wohlfahrt.de
}

\author{
Das Recht auf Teilhabe aller Menschen an der Gesellschaft \\ ist ein wesentliches Ziel des Sozialgesetzbuches und \\ der UN-Behindertenrechtskonvention. Mit der Frage, \\ wie man Teilhabe insbesondere von Menschen mit \\ Behinderung messen - und damit steuern - kann, \\ beschäftigt sich ein gemeinsames Vorhaben der \\ Wohlfahrtsverbände in der Bundesrepublik Deutschland.
}

»Teilhabe heißt nicht, dass jeder Kirschen pflücken darf, sondern dass der Zwerg eine Leiter bekommt.«Das war eine der Rückmeldungen auf der Fachtagung am 2. Mai 2012 in Berlin, auf der das Projekt »Wie misst man Teilhabe in der Eingliederungshilfe? « vorgestellt wurde. Ja, die Leiter ist eine Lösung, doch ist vorab zu klären, ob der Zwerg auch wirklich Kirschen pflücken möchte und nicht Erdbeeren, Birnen oder etwas ganz anderes.

Etwas verkürzt ausgedrückt geht das Projekt mit dem langen Untertitel »Entwicklung, Erprobung und Evaluation von Indikatoren und Instrumenten zur Messung und Beurteilung der Teilhabe von Menschen mit geistigen und/oder Mehrfachbehinderungen oder psychischer Erkrankung « dieser Thematik nach.

\section{Die Projektidee}

Die Verbände der Freien Wohlfahrtspflege beschäftigen sich bereits seit einigen Jahren mit der Frage der Messbarkeit von Teilhabe. Als Ergebnis eines gemeinsamen Arbeitsprozess ist das dreijährige - von der Aktion Mensch geförderte - Projekt am 1. Juni 2011 an den Start gegangen.

Den ersten Anstoß für das Projekt gab eine Veranstaltung der Bundesarbeitsgemeinschaft der Freien Wohlfahrtspflege im September 2007 zum Thema »Qualitätsentwicklung in der Behindertenhilfe und Psychiatrie«.
Die Einführung der UN-Behindertenrechtskonvention und die daraus resultierenden Vorschläge zur Reform der Eingliederungshilfe - insbesondere der mit dem Inklusionsbegriff verbundene Paradigmenwechsel - beförderten die Diskussion um die kritische Überprüfung der Wirksamkeit von Leistungen mit Blick auf die erreichte Teilhabe.

Alle Spitzenverbände der Freien Wohlfahrtspflege haben aus ihrer Verantwortung für die Qualität von Dienstleistungen und Wege der systematischen Qualitätsentwicklung beschritten und hierfür erhebliche Ressourcen aufgewendet. Basierend auf einem werte- und entwicklungsorientierten Verständnis gehen die Grundlinien ihrer strategischen Qualitätsentwicklung und des Qualitätsmanagements in die gleiche Richtung. Dies mündete in die bereits 2004 vom Vorstand verabschiedete gemeinsame QualitätsmanagementPolitik und der sich infolge daraus abgeleiteten Qualitätsziele der Freien Wohlfahrtspflege. (1)

Als Abkehr von institutionsorientiertem Denken und Hinwendung zu personenorientiertem Handeln sind die Hilfen und Dienstleistungen mit den Menschen und bezogen auf ihre ganz individuellen Bedürfnisse und Möglichkeiten zu entwickeln.

Dabei hat sich die Freie Wohlfahrtspflege zum Ziel gesetzt, die Lebensqualität und das Wohlbefinden der Nutzerinnen und Nutzer (2) in den Fokus zu nehmen. 
In dem aus diesen Überlegungen heraus entstandenen Projekt sollen Indikatoren identifiziert werden, mit deren Hilfe der Erfolg der Teilhabe individuell aus der Perspektive der Nutzer gemessen werden kann. Die Grundidee ist, ein Instrumentarium zu entwickeln, mit dem sich feststellen lässt, ob es eine Passgenauigkeit gibt zwischen den individuellen Zielen und Wünschen nach Teilhabe und den Angeboten und Leistungen im Arbeitsfeld der Behindertenhilfe und Psychiatrie.

\section{Konzeption und Zielstellung}

Das Projekt richtet sich an Menschen mit geistiger Behinderung oder psychischer Erkrankung sowie an Menschen mit Mehrfachbehinderung, die Leistungen der Eingliederungshilfe erhalten. Es ist auf die Bereiche Wohnen und Freizeit ausgerichtet. Die wissenschaftliche Begleitung des Projektes erfolgt über das Institut für Personenzentrierte Hilfen gGmbH in Fulda, Prof. Dr. Petra Gromann sowie ihre wissenschaftliche Mitarbeiterin Anke Brückner.

Innerhalb der Bundesarbeitsgemeinschaft der Freien Wohlfahrtspflege erfolgt die Begleitung des Projektes durch eine Steuerungsgruppe, die sich aus Fachvertretern der Gremien Qualitätsmanagement und Behindertenpolitik zusammensetzt. Darüber hinaus wird der Gesamtprozess durch einen Beirat begleitet, der durch Vertreter der beteiligten Nutzer erweitert werden soll.

Bei der Entwicklung der Projektidee wurde ein umfassendes Verständnis von Teilhabe zugrunde gelegt, das auch Aspekte der Lebensqualität mit einbezieht. Es lässt sich jedoch festhalten, dass Teilhabe nicht eindeutig definiert ist. Es können darunter allgemeingültige Standards innerhalb einer Gesellschaft verstanden werden, die eine objektive Betrachtung zulassen, beispielsweise Kriterien für den Zugang zum Bildungssystem.

Aus der Nutzerperspektive jedoch ist die Definition von Teilhabe zunächst eine subjektive Beschreibung dessen, was bezogen auf die ganz persönliche Lebenssituation wichtig ist. Das bedeutet, dass die jeweiligen Definitionen sehr unterschiedlich sein können. Auch kann es eine Diskrepanz geben zwischen der eigenen Beschreibung und dem, was na- hestehende Personen oder professionelle Helfer als wichtig erachten.

Die Herausforderung bei der Entwicklung des Instrumentariums ist es, ausgehend von der subjektiven Beschreibung der Nutzer, ein Indikatoren Set oder einen »Teilhabeanzeiger" zu identifizieren, der objektivierbar ist.

\section{Beteiligte Akteure}

Durch die modellhafte Erprobung in bundesweit zwanzig Piloteinrichtungen von Diensten der Behindertenhilfe und Psychiatrie mit jeweils vier Menschen mit Behinderung sind die Nutzer von Beginn an einbezogen. Aktuell erfolgt die Auswahl der insgesamt achtzig zu beteiligenden Menschen.

In der weiteren Diskussion um die Zielgruppe wurde auch das Ideal formuliert, Menschen mit schwerer und schwerster Behinderung einzubeziehen, entsprechend des Verständnisses, dass Teilhabe ein für alle gültiges Menschenrecht ist. Hier zeigt sich insofern ein Dilemma, dass auch bei größtmöglicher Verschiedenheit in einer Stichprobe nicht alle Möglichkeiten erfassbar sind. Das Projektvorhaben erfordert ein Mindestmaß an Kommunikationsfähigkeit der zu beteiligenden Nutzer.

Bei diesem Forschungsvorhaben mit unmittelbarem Praxisbezug gilt es zwei Ebenen im Blick zu behalten. Neben der Frage des geeigneten Instrumentes und seiner Indikatoren spielt die Frage der Anwendbarkeit eine ebenso große Rolle.

In einem ersten Schritt sind die Indikatoren herauszufinden. Hier haben die beteiligten Wissenschaftlerinnen durch umfangreiche Recherche bereits erfolgter wissenschaftliche Studien zu dem Thema ermittelte und anerkannte Kriterien und Indikatoren identifiziert. Zugrunde gelegt wurden u. a. verschiedene Lebensqualitäts- sowie Organisationsentwicklungsinstrumente.

Darauf folgt in einem zweiten Schritt die Entwicklung eines geeigneten Instrumentes. Für das Projekt ist die Idee einer »Teilhabekiste « entstanden. Dahinter verbirgt sich ein standardisiertes Kartenset, auf dem sich neben Bildern und Symbolen Vorschläge für Teilhabeindikatoren finden. Diese sollen den Nutzern einen spielerischen Zugang zu den möglichen Indikatoren für ihre persönliche Teilhabedefinition eröffnen.

Die Frage der Anwendung, ein- schließlich der Anwenderschulung, ist bei der laufenden Entwicklung der »Teilhabekiste « immer mit zu berücksichtigen. Daraus resultiert eine Art Spielanleitung, also ein Verfahrensablauf, der die Information, Schulung, Umsetzung und Überprüfung des Vorgehens sowie die Ableitung von Verbesserungen umfasst. Bei der Entwicklung dieses Anwendungsprozesses sind verschiedene wissenschaftliche Methoden und Verfahren der qualitativ partizipatorischen Forschung (Delphi-Methode, Photovoice, PATH-Prozess - vgl. Literaturliste) zugrunde gelegt worden.

\section{Umsetzungsstand und weitere Planung}

Nach knapp einem Jahr sind die erforderlichen Vorbereitungen, die wissenschaftliche Recherche sowie der Aufbau der Projektstrukturen abgeschlossen. Eine erste Version der "Teilhabekiste « wurde entwickelt und in einem Pretest hinsichtlich Verständnis und Anwendung erprobt. Erkenntnisse daraus sind in die Überarbeitung des Instrumentes eingeflossen, so dass nunmehr mit einer zweiten Version die Umsetzung in den Piloteinrichtungen erfolgt. Aus der praktischen Anwendung der »Teilhabekiste" sind erste Erfahrungen in den Implementationsprozess, also in die Anwenderschulung und die Ablaufplanung eingeflossen.

Dieser Implementationsprozess findet von Juni bis Oktober dieses Jahres statt. In die Schulungen werden neben den beteiligten Nutzern auch deren Unterstützer aus den beteiligten Einrichtungen und Dienste beteiligt. Dabei handelt es sich in erster Linie um Bezugsbetreuungskräfte oder Qualitätsbeauftragte.

Hierbei wird nach einem sogenannten Kontrollgruppendesign vorgegangen. Die Hälfte aller Teilnehmer wird in einer Präsenzgruppe intensiv durch die wissenschaftliche Mitarbeiterin sowie den Projektkoordinator begleitet. Hier erfolgen die Begleitung in der Anwendung der »Teilhabekiste « und die Identifikation individueller Teilhabeziele der Nutzer also extern. Bei der anderen Hälfte, der Kontrollgruppe, erfolgt eine angeleitete Begleitung durch die wissenschaftliche Mitarbeiterin und den Projektkoordinator. In dieser Gruppe werden die Unterstützer aus den Einrich- 
tungen und Diensten geschult die Nutzer im Umgang mit dem Instrument zu begleiten. Ziel dieses Vorgehens ist es, herauszufinden, ob - und wenn, in welcher Weise - sich Unterschiede im Umgang mit dem Instrument zeigen, abhängig davon, wer die Nutzer unterstützt.

Orientiert an dem sogenannten PATH-Prozess finden mehrere Treffen mit den Nutzern statt. Zunächst gilt es die »Teilhabekiste « auszuprobieren und herauszufinden, was die Nutzer anfangen möchten. Diese Ergebnisse werden schriftlich festgehalten. Ein Folgetreffen dient dazu, die wichtigsten Teilhabeanzeiger $\mathrm{zu}$ identifizieren. In einem weiteren Treffen wird dann ein »Teilhabeaktionsplan « erstellt, der dann nach festgelegten Zeitspannen zu überprüfen ist. Dabei werden der Umsetzungsgrad sowie förderliche und hinderliche Faktoren ermittelt. Dieses Vorgehen findet über den Zeitraum eines Jahres in mehreren Wellen statt.

Insbesondere um den Menschen gerecht zu werden, die sich nur schwer ausdrücken und ihre persönlichen Vorstellungen und Wünsche nur schwer beschreiben können, kommt die Methode »Photovoice« zur Anwendung. Alle am Pilotprojekt beteiligten Nutzer erhalten eine leicht handhabbare Digitalkamera. Sie werden befähigt und ermuntert mit dieser Kamera Bilder von den Dingen, Menschen, Orten usw. zu machen, die Ihnen besonders wichtig sind und die eine Bedeutung für ihre persönliche Lebenswelt haben. Im Zusammenspiel mit den Indikatoren der »Teilhabekiste« werden diese Bilder in einem festgelegten Verfahren ausgewertet. Die Nutzer werden in die Auswertung einbezogen.

Es gilt auch herauszufinden, ob sich aus der Vielzahl der gewählten Indikatoren Schwerpunkte herausfiltern lassen, also Indikatoren, die von den Nutzern besonders häufig genannt werden.

Erste Zwischenergebnisse des Projektes sollen im Sommer 2013 vorgestellt werden. Der Abschluss des Projektes ist für Ende Mai 2014 vorgesehen.

\section{Bedeutung für die Praxis Sozialer Arbeit}

Hinsichtlich der Bedeutung entsprechender Indikatoren für die Praxis Sozialer Arbeit steht allem voran ein besseres Verstehen des subjektiven Teilhabebegriffs.

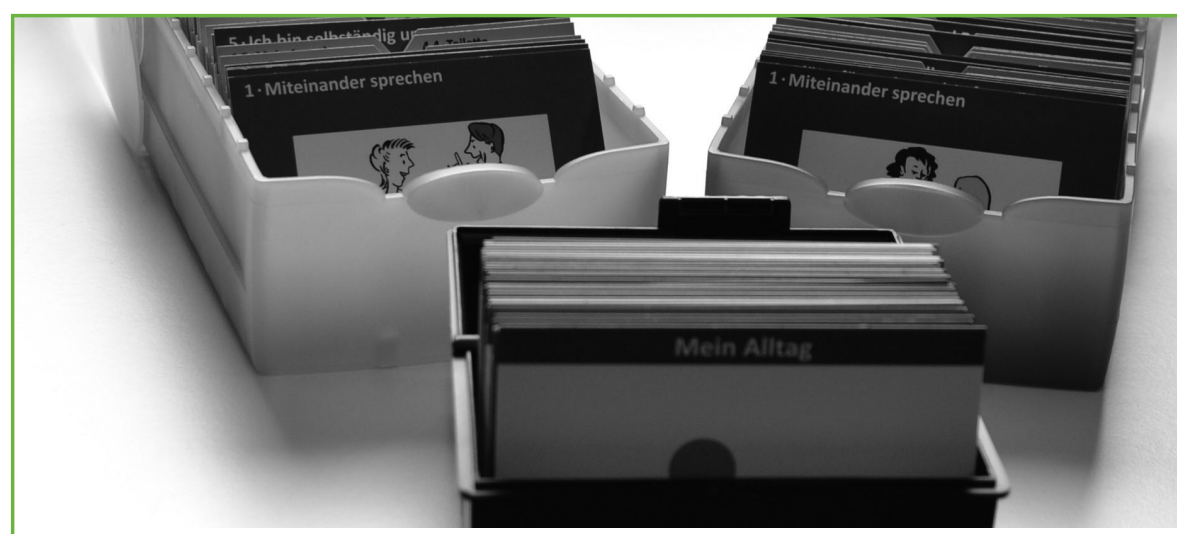

Für das Projekt zur Findung von Kriterien für Teilhabe wurde eine »Teilhabekiste«: ein standardisiertes Kartenset, auf dem sich neben Bildern und Symbolen auch Vorschläge für Teilhabeindikatoren finden. Über den praktischen Einsatz dieses Instrument bei der individuellen Zukunftsplanuung bei Menschen mit Behinderung informiert ein 14-Minuten-Film, der im Internet frei zugänglich angeschaut werden kann.

http://www.youtube.com/watch?v=|3kBWYxd3_Y\&feature=youtu.be

Für die Nutzer heißt dies zunächst eine Begegnung auf Augenhöhe. Sie bekommen den Raum, ihre Vorstellungen zu entwickeln, wie sie leben wollen. Das kann sich sowohl auf bereits Erreichtes als auch neue Ideen beziehen - oder bisher nicht ausgesprochene Träume und Wünsche deutlich machen. Ausgehend davon, dass jeder Mensch Fähigkeiten in sich trägt, sich zu entwickeln und dass die Sehnsucht nach etwas Schönem diese Kräfte mobilisiert, soll die Arbeit mit der »Teilhabekiste « ermöglichen, den Blick darauf zu lenken, was eine gelungene Zukunft ausmachen würde.

Für die Träger dürften die Erkenntnisse insbesondere für das interne Qualitätsmanagement einen hohen Nutzen haben und ermöglichen, bestimmte Strukturen und Prozesse genauer an die Erfordernisse der Nutzer anzupassen. Natürlich ist hier zu bedenken, dass nicht alle Wünsche und Ziele der Nutzer durch die Träger und die Mitarbeitenden zu beeinflussen sind. Die Fokussierung der Nutzerperspektive könnte aber Einfluss auf die Haltung ausüben, was wiederum zu einem Kulturwandel in den Einrichtungen und Diensten und somit zu einer Organisationsentwicklung führen könnte.

Vorstellbar ist, dass die Ergebnisse aus der individuellen Teilhabeplanung in die jeweilige Hilfeplanung einfließen, setzt sie doch vor der Hilfeplanung an und ist stärker auf Sehnsüchte ausgerichtet, die dann erst im Folgenden auf die Realisierbarkeit bestimmter Maßnahmen zu operationalisieren sind.
Mit dem Ende des Projektes werden sich noch keine umfassenden Aussagen ableiten lassen, inwieweit die Leistungen der Eingliederungshilfe anzupassen wären, damit sie stärker mit den Zielen der Nutzer übereinstimmen. Das würde wiederum die Frage der gezielten Finanzierbarkeit einbeziehen. Jedoch bieten das Instrument und der dazugehörige Prozess das Potential genau dieser Frage nachzugehen. Mit einer breiteren Anwendung und dem entsprechenden Monitoring ließen sich hierzu Aussagen treffen.

Es gibt noch eine Reihe weiterer Überlegungen, die möglicherweise aus den Projektergebnissen abzuleiten sind. So könnte eine klarere Definition des Teilhabebegriffs den Weg in ein einheitliches Vorgehen der Leistungsausgestaltung der einzelnen Bundesländer öffnen. Bei erfolgreicher Umsetzung wäre eine Ausdehnung auf weitere Bereiche oder weitere Nutzergruppen (z. B. Altenhilfe) vorstellbar, ebenso die Vernetzung mit weiteren inklusiven Projekten (z.B. NUEVA). Nicht zuletzt eröffnet das Projekt durch seinen Perspektivenwechsel einen weiteren Schritt in eine inklusive Gesellschaft.

\section{Anmerkungen}

(1) Siehe Internet www.bagfw-qualitaet.de/Grundsätze/.

(2) Zur besseren Lesbarkeit wird im Folgenden im Plural von Nutzern gesprochen. Gemeint sind immer beide Geschlechterformen. 